Int. J. Dev. Biol. 57: 829-836 (2013)

doi: $10.1387 /$ ijdb.130109sc

\title{
Essential role of AWP1 in neural crest specification in Xenopus
}

\author{
JEONG-HAN SEO ${ }^{1}$, DONG-SEOK PARK ${ }^{1}$, MINA HONG ${ }^{1}$, EUN-JU CHANG ${ }^{1}$ and SUN-CHEOL CHOI ${ }^{* 1,2}$ \\ ${ }^{1}$ Department of Biomedical Sciences, Cell Dysfunction Research Center (CDRC) and ${ }^{2}$ Department of Biochemistry and \\ Molecular Biology. University of Ulsan College of Medicine, Pungnap-Dong, Songpa-Gu, Seoul, Republic of Korea
}

\begin{abstract}
The neural crest (NC) comprises a transient and multipotent embryonic cell population, which gives rise to a wide variety of cell types, including craniofacial cartilage, melanocytes, and neurons and glia of the peripheral nervous system. The NC is induced by the integrated action of Wnt, FGF, and BMP signaling, and its cell fates are subsequently specified by a genetic cascade of specific transcription factors. Here we describe a critical role of AWP1 in NC induction during Xenopus early development. Xenopus AWP1 (XAWP1) was found to be expressed in the presumptive preplacodal ectoderm, neural tissue, and posterior dorsal mesoderm, but was absent in the neural fold along the anterior-posterior axis of the neurulae. Notably, XAWP1 was induced by FGF8a in naïve ectodermal tissue. XAWP1-depleted embryos exhibited defects in pigmentation, craniofacial cartilage, and in the dorsal fin. A knockdown of XAWP1 impaired both endogenous and the FGF8a or Wnt8-induced expression of NC markers without affecting mesoderm formation. Furthermore, NC induction inhibited by XAWP1 depletion was rescued by co-expression of activating forms of $\beta$-catenin or TCF3. In addition, overexpression of XAWP1, in concert with BMP inhibition, induced the expression of neural plate border specifiers, Pax3 and Msx1, and these regulatory factors recovered NC induction in the XAWP1-depleted embryos. $\beta$-catenin stability and Wnt-responsive reporter activity were also impaired in AWP1-depleted cells. Taken together, these results suggest that XAWP1 functions as a mediator of Wnt signaling to regulate NC specification.
\end{abstract}

KEY WORDS: AWP1, neural crest induction, Wnt, Xenopus

\section{Introduction}

The neural crest (NC) is a vertebrate multipotent embryonic cell population, which arises at the border between the neural plate and epidermis along the antero-posterior (AP) axis posterior to the diencephalon and migrates to several parts of the embryo to differentiate into a large variety of cell types, including melanocytes, craniofacial cartilage and neurons and glia of the peripheral nervous system (Le Douarin and Dupin, 2003; Sauka-Spengler and Bronner-Fraser, 2008). During gastrulation, NC cells are induced by the combined action of Wnt, fibroblast growth factor (FGF), bone morphogenetic protein (BMP), and Notch signals that originate from the epidermis, neural plate, and underlying mesoderm (Sauka-Spengler and Bronner-Fraser, 2008; Steventon et al., 2005). These NC-inducing signals are integrated at the neural plate border (NPB) to activate a genetic cascade of transcription factors, first inducing the expression of NPB specifiers (Msx1, Pax3, Zic1, Ap2, Dlx3) that, in turn and in concert with the same signaling pathways, trigger the expression of NC specifiers (Sox9, Sox10, Slug, FoxD3) (Heeg-Truesdell and LaBonne, 2004; Prasad et al., 2012; Sauka-Spengler and Bronner-Fraser, 2008). NC specifiers ultimately control NC behaviors such as the epithelial to mesenchymal transition (EMT), delamination, migration and differentiation by regulating the adhesive properties, shape, motility, cell-cycle, cell survival and multipotency of NC progenitors (Sauka-Spengler and Bronner-Fraser, 2008; Theveneau and Mayor, 2012). However, our current understanding of the complex gene regulatory network for NC specification, migration and differentiation into diverse derivatives appears to be preliminary. More connections and the hierarchical organization of the transcription factors involved in NC formation remain to be elucidated.

Canonical Wnt signaling regulates cell fates and cell prolifera-

Abbreviations used in this paper: $\mathrm{BMP}$, bone morphogenetic protein; FGF, fibroblast growth factor; $\mathrm{MO}$, morpholino oligo; $\mathrm{NC}$, neural crest; $\mathrm{NPB}$, neural plate border; ODC, ornithine decarboxylase; XAWP1, Xenopus AWP1

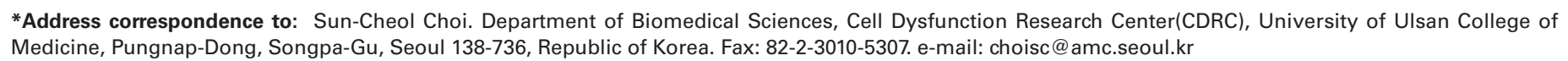


tion through $\beta$-catenin/TCF complex-dependent gene transcription (MacDonald et al., 2009). A large body of evidence suggests that canonical Wnt/ $\beta$-catenin signaling is implicated in NC induction and subsequent development (Stuhlmiller and Garcia-Castro, 2012). Double-homozygous null mutant mice for Wnt1/Wnt3ashow severe abnormalities in NC derivatives (Ikeya et al., 1997). In Xenopus and zebrafish, the morpholino-mediated knockdown of Wnt8 or Wnt3ainhibits the expression of NPB and NC markers (Elkouby et al., 2010; Hong et al., 2008; Lewis et al., 2004). In addition, Wnt6, which is secreted from the chick non-neural ectoderm adjacent to the NPB, is necessary and sufficient to induce NC cells in avian embryos (Garcia-Castro et al., 2002). Gain- and loss-of-function analyses of other Wnt signaling components, including $\beta$-catenin, Frizzled7, LRP6, Dishevelled, and Kremen2, further support the model that NC formation involves the canonical Wnt pathway (Abu-Elmagd et al., 2006; Hassler et al., 2007; Tamai et al., 2000).

In this study, we have identified AWP1 as a critical regulator of NC specification in Xenopus early embryogenesis. AWP1 (also known as zinc finger AN1 type-6; ZFAND6) was originally identified as a protein associated with the serine/threonine kinase PRK1, a lipid-activated kinase belonging to the protein kinase C superfamily (Duan et al., 2000). AWP1 contains A20 and AN1 zinc finger domains at its $\mathrm{N}$ - and $\mathrm{C}$-terminal, respectively, and is known as ubiquitin-binding negative modulator of NF-kB activity (Fenner et al., 2009). Recently, AWP1 has also been shown to affect apoptotic cell death as a regulator of NF-kB activity (Chang et al., 2011) and protein export during biogenesis of the peroxisome, a subcellular organelle (Miyata et al., 2012). AWP1 is expressed in several human tissues and also in the early stages of mammalian development (Duan et al., 2000), but its physiological function remains unknown. We have found that AWP1 affects the expression of NPB and NC markers in the neural fold by mediating $\mathrm{Wnt} / \beta$-catenin signaling. Notably, it is a target gene of FGF signaling and is also essential for NC induction via this pathway. Our present results thus suggest that AWP1 functions to mediate the crosstalk of Wnt and FGF signaling in NC induction.

\section{Results}

\section{Expression pattern of AWP1 in early Xenopus embryogenesis}

To investigate the biological function of $A W P 1$ in vertebrates, we first observed its developmental expression pattern in Xenopus laevis embryos. Temporally, XAWP1 shows both maternal and zygotic transcription in early embryogenesis as revealed by RTPCR analysis (Fig. 1A). We also performed in situ hybridization to examine its spatial expression pattern. At the early cleavage stages, XAWP1 maternal transcripts are localized to the animal hemisphere of the embryo (Fig. 1B), and this pattern persists into the blastula stages (data not shown). As gastrulation proceeds, its expression is observed in the mesoderm and overlying ectoderm (Fig. $1 \mathrm{C}, \mathrm{D}$ ). During neurulation, its zygotic message is detectable in the presumptive preplacodal ectoderm and neural tissue but not in the anterior and lateral neural folds (Fig. $1 \mathrm{E}-\mathrm{G}$ ). Of note, $X A W P 1$ is strongly expressed in the posterior dorsal mesoderm in neurulae (Fig. $1 \mathrm{E}, \mathrm{H}$ ). At the tadpole stages, its specific expression is found in the eyes, as well as in NC derivatives such as branchial arches and otic vesicle in the head region, and in mesodermal derivatives such as somites and kidney tubules along the trunk region of embryo (Fig. 1I). In addition, we examined whether XAWP1 expression could be induced by signals critical for Xenopus development, including FGF, Wnt and Nodal (Kimelman, 2006). Intriguingly, RT-PCR analysis showed that injection of FGF8a RNA, but not Wnt8 and Xnr-1, could enhance XAWP1 expression in the naïve ectodermal tissue (Fig. 1J), suggesting a possible role of XAWP1 in developmental events involving FGF signaling.

Fig. 1. Expression of the XAWP1 gene. (A) RT-PCR analysis showing the temporal expression pattern of AWP1 in Xenopus early development. Ornithine decarboxylase (ODC) serves as a loading control. $-R T$, a stage 27 control embryo in the absence of reverse transcriptase. (B-I) Spatial expression pattern of XAWP1. (B) Animallateral view with the vegetal pole to the bottom. (C) Vegetal-lateral view with dorsal to the right. (D) Sagittal section of a stage 10.5 gastrulae. Arrows in $(C, D)$ denote the dorsal blastopore lip. Arrowheads indicate the involuting dorsal mesoderm. (E) Dorsal view with anterior to the top. (F) Anterior view of the embryo shown in (E) with dorsal to the top. ne, neural ectoderm; pe, preplacodalectoderm. $(\mathbf{G}, \mathbf{H})$

A
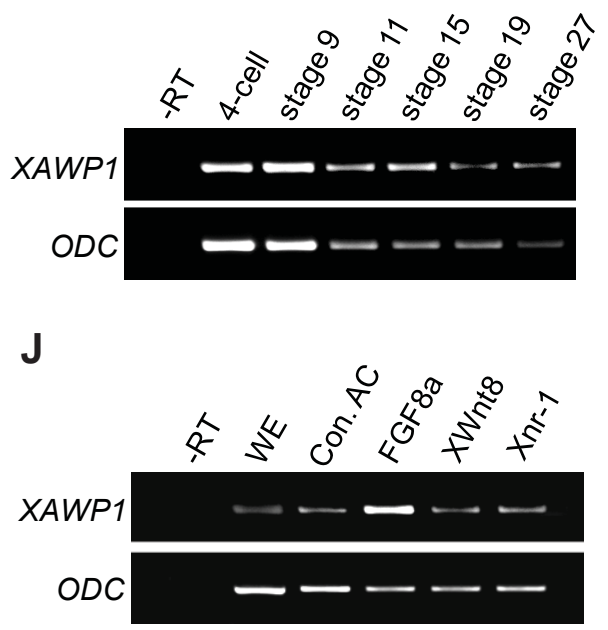

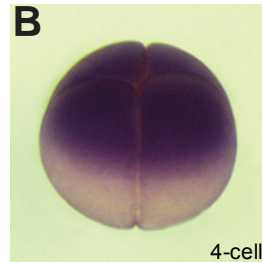

E
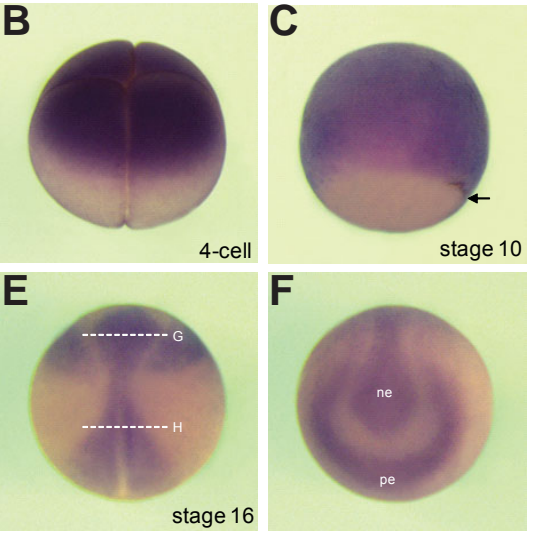

$\mathbf{F}$
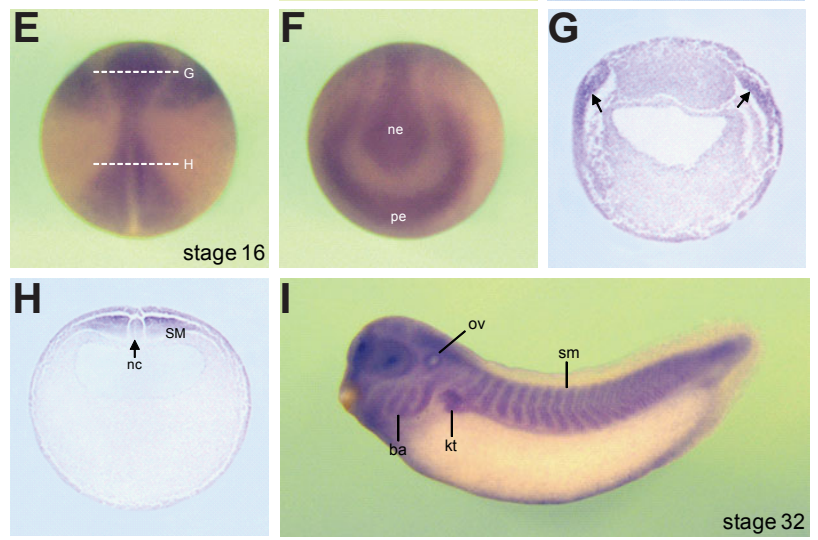

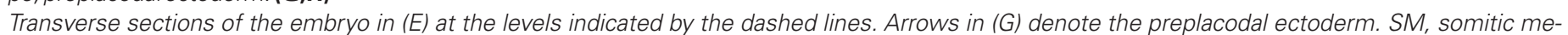

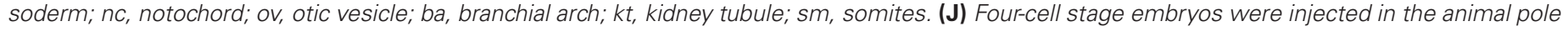

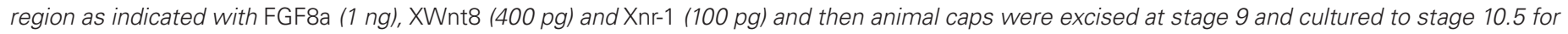
RT-PCR analysis. WE, a stage 10.5 whole embryo. Con. AC, uninjected control animal caps. 
Fig. 2. A knockdown of XAWP1 disrupts neural crest derivatives. (A) Western blotting analysis of the specificity and efficiency of MOs. Four-cell stage embryos were injected in the animal pole region with XAWP1-Myc (200 pg) alone or with Co MO 130 ng), AWP1 MO1 (20 ng) or AWP1 MO2 (30 ng) and animal cap explants were then dissected at stage 9 and cultured to stage 11 for western blotting with an anti-myc antibody. $\beta$-actin was used as a loading control. (B-G) Embryos were injected in the dorso-animal region of two blastomeres at the 8-cell stage with Co MO (10 ng), AWP1 MO1 (10 $\mathrm{ng}$ for $C$ and D, $5 \mathrm{ng}$ for $E$ and G), AWP1 MO2 (30 ng) and hAWP1 (5 pg) as indicated and cultured to stage 38. Arrows and arrowheads indicate defects in the dorsal fin and pigmentation, respectively. sub, suboptimal. $\mathbf{( H , I ) ~ F o u r - ~}$ A

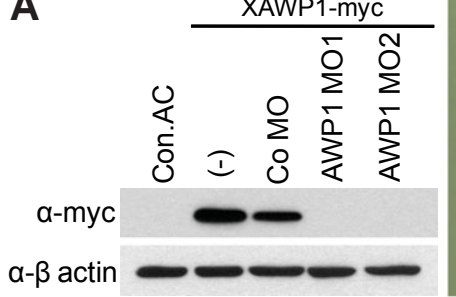

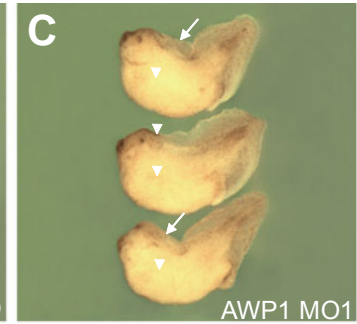

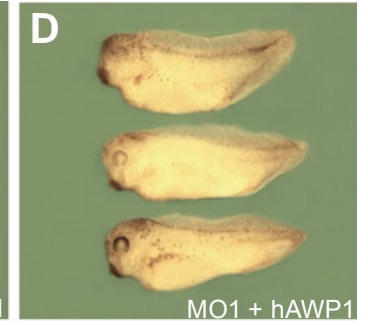

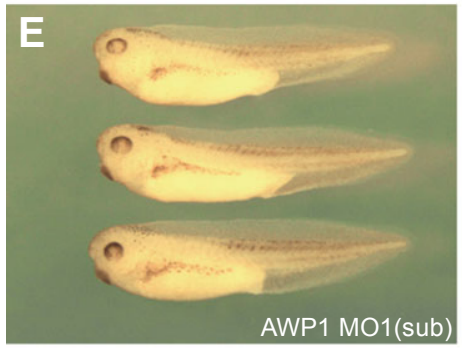

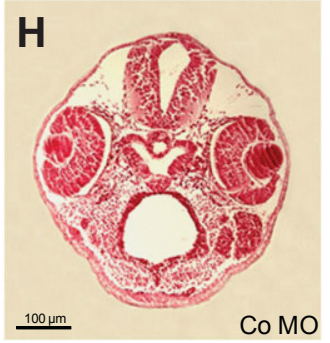

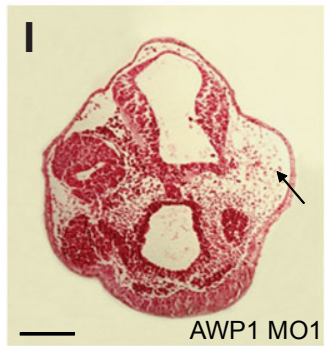
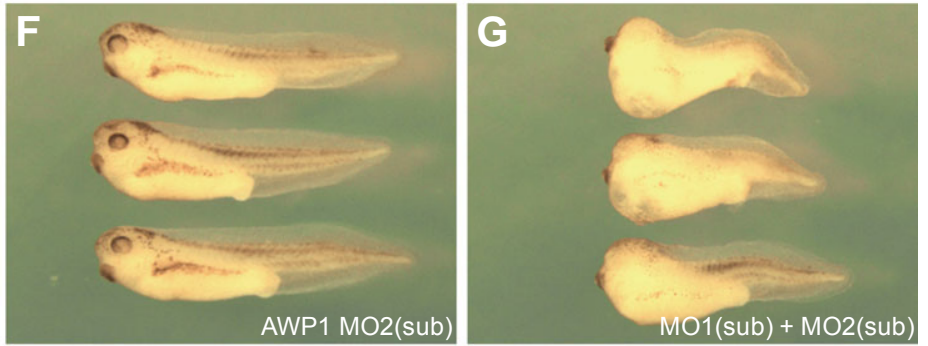

J

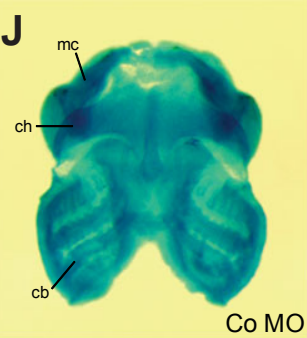

K

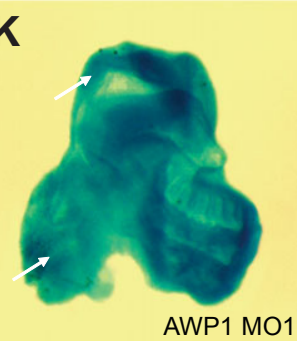

cell stage embryos were injected in the dorso-animal region of one blastomere with AWP1 MO1 (5 ng) or Co MO (10 ng) and then subjected to paraffin sectioning and eosin staining at stage 40. An arrow indicates the defective eye in the injected side. (J,K) One blastomere of a four-cell stage embryo was injected in the dorso-animal region with AWP1 MO1 (5 ng) or Co MO (10 ng) and then stained with alcian blue at stage 45. Arrows indicate severely disrupted ceratobranchial and Meckel's cartilage in the injected side. cb, ceratobranchial cartilage; mc, Meckel's cartilage; ch, ceratohyal cartilage.

\section{Knockdown of XAWP1 causes defective neural crest deriva- tives}

We employed an anti-sense morpholino oligo (MO)-mediated knockdown approach for loss-of-function analysis of XAWP1. For this, we designed two different MOs (MO1 and MO2), which target distinct sequences around the translation initiation site of XAWP1 mRNA. BLAST searches against XenopusEST database revealed that several genes have internal nucleotide substitutions, possibly due to the allotetraploidy, displaying over $93 \%$ identity with XAWP1 . As the 5' regions around the transcription initiation sites of these genes are highly conserved (data not shown), most of them appear to be targeted efficiently by the two MOs. Western blotting also demonstrated that both AWP1 MO1 and MO2, but not control (Co) $\mathrm{MO}$, could efficiently inhibit the production of XAWP1 protein (Fig. 2A). To examine the morphological phenotypes of XAWP1-depleted embryos, we targeted the injection toward the dorsal region of embryos. As shown in Fig. 2C, AWP1 MO1-injected embryos exhibited severe defects in craniofacial structures such as the eyes, and in pigmentation and fin formation along the trunk region of embryos $(89 \%, \mathrm{n}=68)$, and this malformation could be partially rescued by co-injection of human AWP1 ( $h A W P 1$ ) RNA (Fig. 1D, 58\%, $\mathrm{n}=73$ ), which has no the target sequence of the $\mathrm{MO}$ and appears to be resistant to its inhibitory effect. To further confirm the specificity of these MO phenotypes, we also investigated whether the two MOs could synergize to impair the formation of craniofacial struc- tures. Thus, we titrated the MOs down to levels that did not yield the phenotypes on their own. Targeted injection of either MO1 or $\mathrm{MO} 2$ at such suboptimal levels did not elicit any defects (Fig. 2E, F), whereas co-injection of the MOs at the same reduced doses interfered with head formation and pigmentation $(74 \%, n=72$; Fig. $2 \mathrm{G})$, phenocopying the defective embryos that were injected with a high level of MO1 alone. These results thus corroborate the specific effects of the MOs in disrupting the craniofacial structures. In addition, histological analysis clearly showed that injection of AWP1 MO1, but not Co MO, could inhibit formation of the lens and multilayered retina in the eye and of head mesenchyme in the injected side of the embryo (Fig. $2 \mathrm{H}, \mathrm{I}$ ). We further examined the morphology of craniofacial cartilage, a NC derivative, in XAWP1depleted embryos. As visualized by alcian blue staining, unilateral injection of AWP1 MO1 resulted in a severe loss of craniofacial skeletal elements, including Meckel's, ceratobranchial and ceratohyal cartilage in the injected side compared with the uninjected control side of embryo (Fig. 2K). In contrast, injection of Co MO had no effect on the size or morphology of the craniofacial cartilage (Fig. 2J). Taken together, these data suggest that XAWP1 plays a critical role in the formation of NC-derived structures.

\section{XAWP1 is required for neural crest specification}

We next investigated the effects of depleting XAWP1 on the initial steps of NC formation. Targeted injection of AWP1 MO1, but 
not Co MO, abrogated the expression of the NC markers, Sox10 and Slug in the injected side, compared with the uninjected side of embryos ( $86 \%, n=50$ for Sox $10 ; 65 \%, n=65$ for Slug; Fig. 3 A,B, $\mathrm{D}, \mathrm{E})$. These repressive effects of the $\mathrm{MO}$ were rescued to some degree by co-expression of $h A W P 1$ (54\%, $n=58$ for Sox $10 ; 67 \%$, $\mathrm{n}=70$ for Slug; Fig. $3 \mathrm{C}, \mathrm{F})$. Moreover, while single injection of MO1 or $\mathrm{MO} 2$ at below threshold levels did not affect the expression of Sox 10(Fig. $3 \mathrm{G}, \mathrm{H}$ ), the co-injection of the MOs at the same reduced concentrations could efficiently repress its expression in the injected side ( $40 \%, n=45$; Fig. $3 \mathrm{I})$. This confirmed the specificity of the MOs in inhibiting NC specification. NC markers are induced ectopically in animal cap tissue by co-expressing Wnts and BMP inhibitors such as noggin and chordin (Chang and Hemmati-Brivanlou, 1998). We also used this animal cap assay to test whether XAWP1 is required for ectopic NC induction. Co-injection of Wnt8 and noggin RNAs induced the expression of Slug and Sox9 in the animal cap tissues (Fig. 3J). MO1 or MO2-mediated knockdown of XAWP1 could down-regulate this ectopic expression, whereas the co-injection of Co MO had no effect (Fig. 3J). These results thus suggest that $X A W P 1$ is essential for the early specification of NC cells. As XAWP1 is expressed in the mesoderm at the gastrula and neurula stages as described above, we next examined whether it might be involved in mesoderm induction and/or maintenance. Although the XAWP1-depleted embryos exhibited the seemingly disrupted trunk structure as shown above, its knockdown had no effect on the expression of an early pan-mesodermal marker, Xbraand late mesodermal markers, MyoD and Xnot, suggesting that it is not critical for mesoderm formation.

\section{XAWP1 acts upstream of neural plate border specifiers}

The expression of NPB specifiers such as Msx 1 and Pax3, which function as early factors in the NC genetic cascade, is regulated by Wnt and FGF signals (Monsoro-Burq etal., 2005). We next asked whether XAWP1 could also control the expression of these NPB markers. To address this, we performed the animal cap assay described above in which NC and NPB markers can be induced by Wnt or FGF signals combined with BMP inhibition. Notably, injection of increasing levels of XAWP1 RNA could induce the expression of these NPB markers as well as of a NC marker, FoxD3 in the animal caps (Fig. 4A) in the presence of a dominant negative (DN) BMP4 receptor, which inhibits BMP signaling, suggesting a possible role for AWP1 as a mediator of Wnt and/or FGF signaling. Thus, we further examined whether Msx1 and Pax3 might function downstream of XAWP1 to regulate NC specification. The targeted injection of AWP1 MO1 impeded the expression of the marker Sox 10 in the injected side of the embryo (59\%, n=55; Fig. 4B), which could be efficiently rescued by co-injection of Msx1 or Pax3 RNA (77\%, $\mathrm{n}=52$ for $M s \times 1 ; 85 \%$, $\mathrm{n}=50$ for Pax3; Fig. 4 C,D). Taken together, these data indicate that $X A W P 1$ acts upstream of NPB regulators to specify NC cells.

\section{XAWP1 mediates neural crest induction by Wnt and FGF signaling}

Wnt/ $\beta$-catenin and FGF signals have been shown to be crucial for NC induction (Stuhlmiller and Garcia-Castro, 2012). We examined whether NC induction by these signals depends on XAWP1 activity. The unilateral injection of FGF8a RNA strongly enhanced the expression of Sox 10 along the neural fold in the injected side of the embryo (74\%, $\mathrm{n}=60$; Fig. 5A), and co-injection of AWP1 MO1, but not $\mathrm{Co} \mathrm{MO}$, could impair this expansion (93\%, n=45; Fig. 5B,
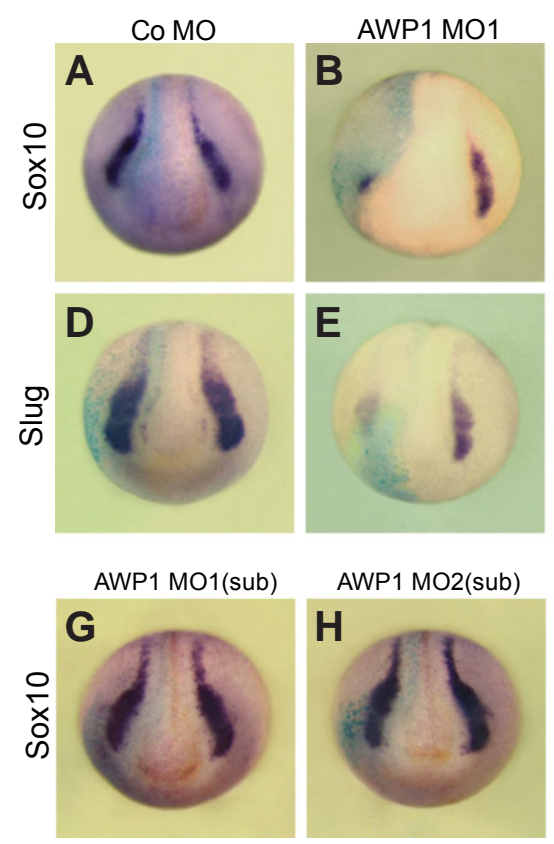

AWP1 MO2(sub)
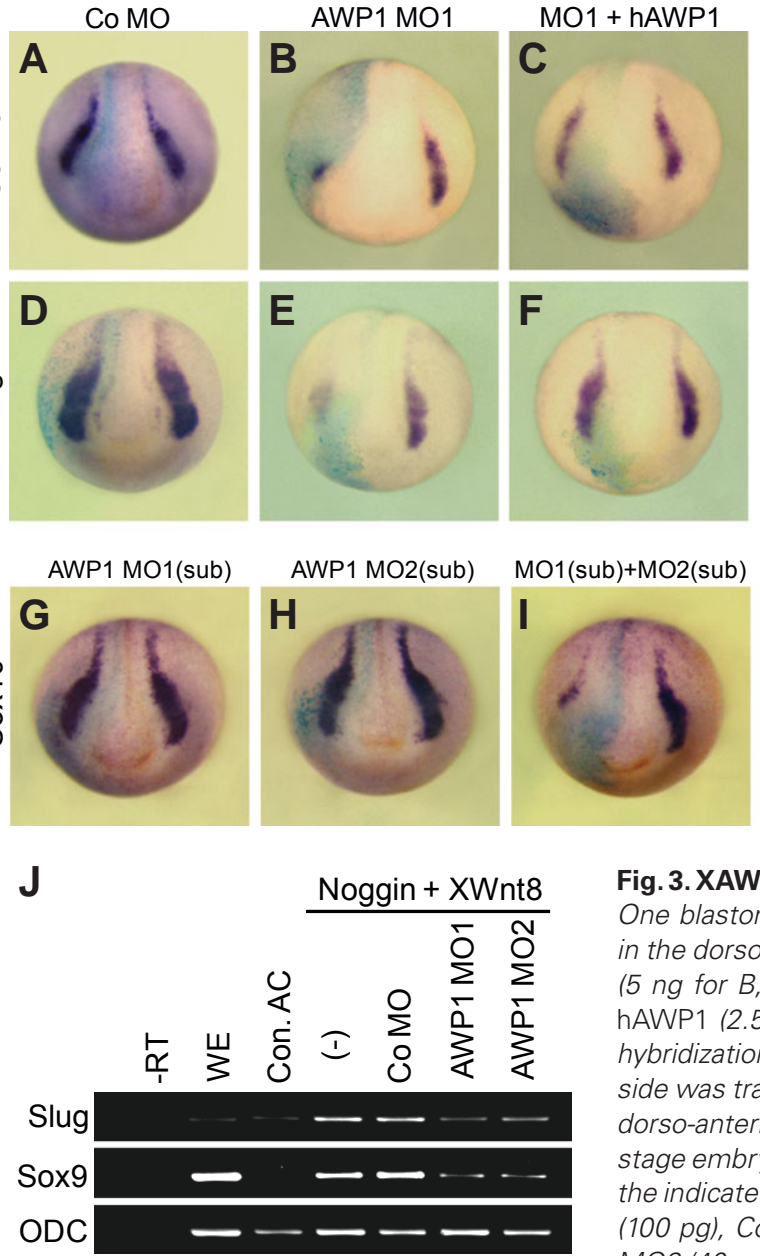

Fig. 3. XAWP1 is required for neural crest induction. (A-I) One blastomere of a four-cell stage embryo was injected in the dorso-animal region with Co MO (5 ng), AWP1 MO1 (5 $n g$ for B,C,E,F; 2 ng for G,I), AWP1 MO2 (20 ng) and hAWP1 $(2.5 \mathrm{pg})$ as indicated and then subjected to in situ hybridization against Slug or Sox10 at stage 16. The injected side was traced by lacZ staining. All embryos are shown in dorso-anterior view with posterior to the top. (J) Four-cell stage embryos were injected in the animal pole region with the indicated combination of noggin (100 pg), XWnt8-CSKA (100 pg), Co MO (40 ng), AWP1 MO1 (20 ng) and AWP1 MO2 (40 ng), and the animal caps were dissected at stage 9 and cultured to stage 16 for RT-PCR analysis. (K-P) Two blastomeres of a four-cell stage embryo were injected in the dorsal-marginal region with Co MO (10 ng) or AWP1 MO1 (10 ng) and subsequently subjected to in situ hybridization against Xbra at stage 10.5 or MyoD or Xnot at stage 14. Embryos are shown in vegetal view with dorsal to the top $(\mathbf{K}, \mathbf{L})$ or in dorsal view with anterior to the top (M-P). 
A
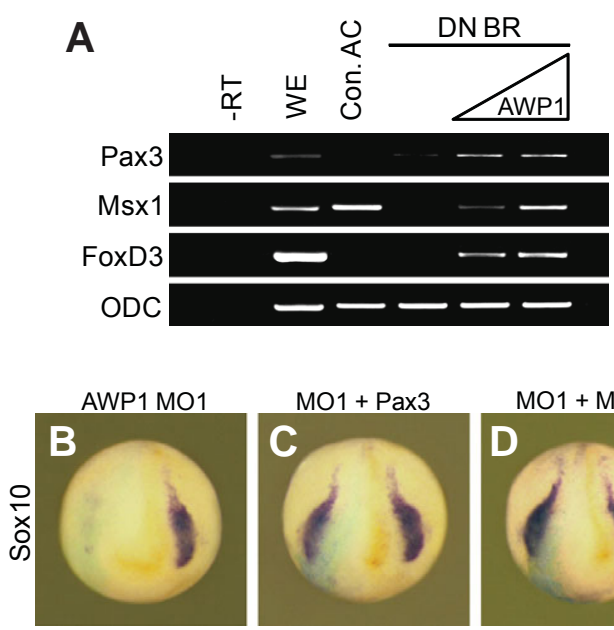

$\mathrm{MO} 1+\mathrm{Pax} 3$
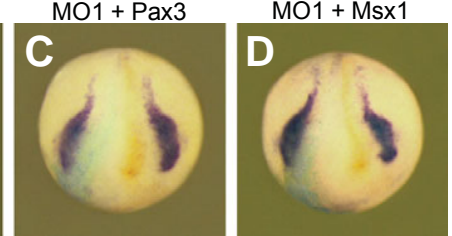

Fig. 4. XAWP1 regulates neural crest induction upstream of Msx1 and Pax3. (A) Four-cell stage embryos were injected in the animal pole region with DN BMP4 receptor (1 ng) with or without XAWP1 $(0.5,2 \mathrm{ng})$, and the animal cap explants were excised at stage 9 and cultured to stage 11 for RT-PCR analysis. (B-D) Four-cell stage embryos were injected in the dorso-animal region of one blastomere with AWP1 MO1 (5 ng) with or without Msx1 (100 pg) or Pax3 (70 pg) as indicated and then subjected to in situ hybridization against Sox10 at stage 16.

C), suggesting a critical role of XAWP1 in FGF8a induction of the NC. In addition, injection of $X W n t 8$-expressing plasmid resulted in ectopic rostral expansion of Sox 10 in the injected side $(62 \%, \mathrm{n}=71$; Fig. 5D), which could be potently inhibited by co-injection of $A W P 1$ MO1 (89\%, n=60) but not by Co MO (Fig. 5 E,F). Similarly, targeted expression of a DN XWnt8RNA strongly abrogated the expression of Sox 10 along the lateral neural ridge $(71 \%, n=68$; Fig. $5 \mathrm{G})$ and this suppression could be rescued by co-expressing XAWP1 RNA $(55 \%, \mathrm{n}=87$; Fig. $5 \mathrm{H})$. Furthermore, the AWP1 MO1-mediated repression of Sox 10 expression ( $61 \%, \mathrm{n}=83$; Fig. 5I) could be reverted to normal levels by co-injecting an activating form of $\beta$-catenin ( $p t$ $\beta$-catenin) or of Tcf3 (VP16-Tcf3) RNA ( $67 \%, \mathrm{n}=87$ for $p t \beta$-catenin; $65 \%, \mathrm{n}=83$ for VP16-Tcf3; Fig. $5 \mathrm{~J}, \mathrm{~K})$. pt $\beta$-catenin is a stabilized mutant of $\beta$-catenin that lacks the phosphorylation sites required for its degradation, and expression of $p t \beta$-catenin or VP16-Tcf 3 is able to induce strongly the transcription of Wnt-responsive genes in the nucleus (MacDonald et al., 2009). Thus, these data suggest that XAWP1 controls NC induction downstream of Wnt8 ligand and upstream of the $\beta$-catenin/Tcf3 complex in the Wnt signaling pathway. We conclude from this evidence that XAWP1 functions to mediate the activities of Wnt and FGF signaling in NC induction.

\section{XAWP1 affects the stability of $\beta$-catenin}

Wnt/ $\beta$-catenin signaling regulates gene transcription by controlling the stability of $\beta$-catenin protein (MacDonald et al., 2009). Since XAWP1 regulates NC specification by acting in a position between a Wnt ligand and the $\beta$-catenin/Tcf3 complex in the Wnt signaling cascade as shown above, we tested whether it could affect the stability of $\beta$-catenin. Interestingly, injection of AWP1 $\mathrm{MO} 1$, but not $\mathrm{Co} \mathrm{MO}$, reduced the level of $\beta$-catenin-myc protein, which could be reversed by co-expression of hAWP1 RNA (Fig. $6 \mathrm{~A})$. Furthermore, co-injection of XAWP1 up-regulated slightly its level (Fig. 6A). These results suggest that XAWP1 plays a critical role in the stabilization of $\beta$-catenin. Next, we performed reporter assays to confirm the requirement of AWP1 for Wnt/ $\beta$-catenin signaling. The expression of a constitutively active form of Wnt receptor, LRP6 ( $\triangle N L R P 6)$ or Dishevelled increased the luciferase activity driven by Wnt-responsive elements in a TOPFlash reporter gene (Fig. 6B). However, the siRNA-mediated knockdown of AWP1 could interfere with the full activation of this reporter by the Wnt signaling components, although a control siRNA had a marginal effect on reporter activation (Fig. 6B). Overall, these results suggest that $A W P 1$ can affect Wnt signaling by modulating the stability of $\beta$-catenin.

\section{Discussion}

Although AWP1 has been shown to be expressed in mouse and human early embryos as well as in several human tissues (Duan et al., 2000), its physiological function remains unknown. In this study, we have identified the pivotal role of AWP1 in NC formation during vertebrate early development. In Xenopus neurulae, XAWP1 is absent in the neural fold, a neural crest-forming region but is detectable in its surrounding tissues, including the
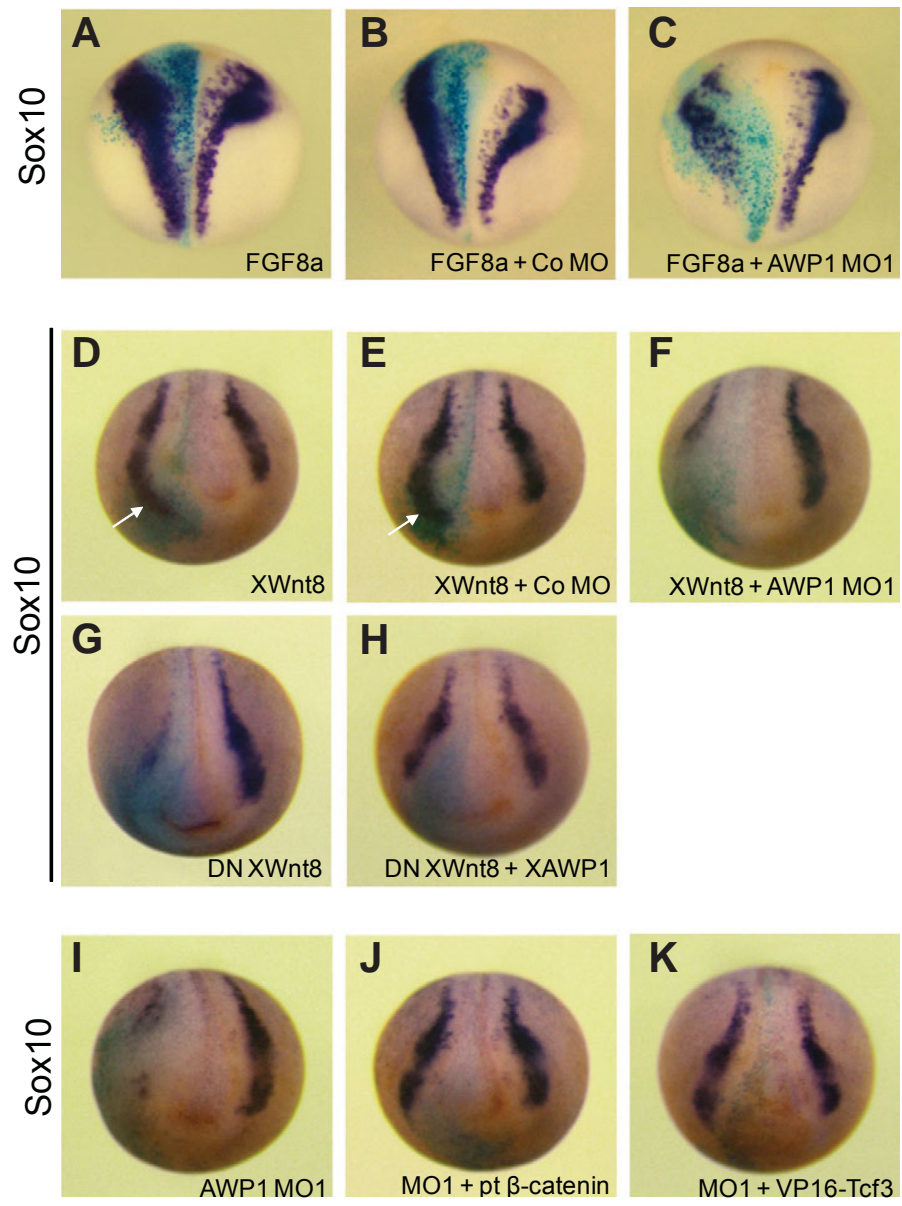

Fig. 5. Neural crest induction by FGF or Wnt signal requires XAWP1 function. (A-K) One blastomere of a eight-cell stage embryo was injected in the dorso-animal region as indicated with FGF8a (20 pg), XWnt8-CSKA (100 pg), DN XWnt8 (400 pg), XAWP1 (300 pg), pt $\beta$-catenin (100 pg), VP16Tcf3 (50 pg), Co MO (5 ng) and AWP1 MO1 (5 ng) and then subjected to in situ hybridization against Sox10 at stage 16. Arrows indicate the expanded expression of Sox 10 . 
preplacodal ectoderm, neural ectoderm, and axial and paraxial mesoderm (Fig. 1). This expression pattern suggests that XAWP1 affects neural crest induction non-autonomously in the adjacent tissues but not in the neural crest progenitors. In support of this, the paraxial somitic mesoderm where the strong expression of $X A W P 1$ is visible, has been shown to be a key source of neural crest inducing signals such as Wnt and FGF in Xenopus (Hong et al., 2008; Monsoro-Burq etal., 2003). Furthermore, XAWP1 acts as a mediator of Wnt and FGF signaling to specify neural crest cells as shown in this work. In contrast, XAWP1 appears not to be critical for mesodermal specification as evidenced by no changes in the expression of early and late mesodermal markers in the XAWP1depleted embryos (Fig. 3), which display the seemingly defective trunk structure. In addition to the marked expression of XAWP1 in the preplacodal ectodermal region, the craniofacial defects caused by its knockdown, in particular, the malformed eyes, suggest that preplacodal development as well as neural crest formation is also disrupted in the XAWP1-depleted embryos. The neural crest cells can organize the vertebrate eye by contributing to the alignment

A

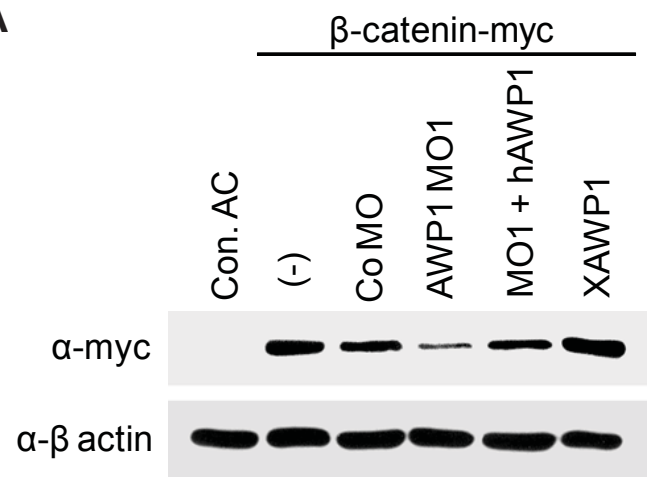

B

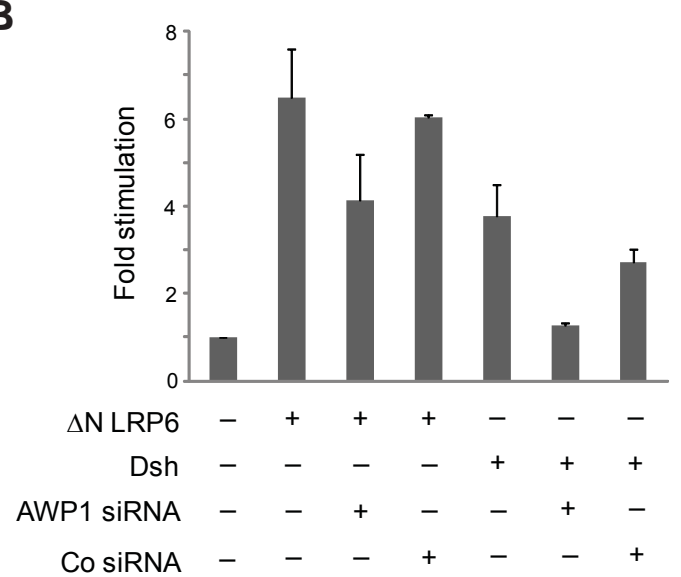

Fig. 6. AWP1 is involved in Wnt/ $\beta$-catenin signaling. (A) Four-cell stage embryos were injected in the animal pole region with the indicated combinations of $\beta$-catenin-myc (100 pg), Co MO (40 ng), AWP1 MO1 (40 ng), hAWP1 (400 pg) and XAWP1 (400 pg), and the animal caps were excised at stage 9 and cultured to stage 11 for western blotting. (B) HEK 293T cell were transfected as indicated with $\triangle$ N LRP6 (450 ng), XDsh (450 ng), AWP1 siRNA (50 nM) and Co siRNA (50 nM). Three independent experiments were performed and a single representative result is shown. Error bars denote standard deviations. of retina and lens via TGF- $\beta$ and Wnt signaling (Grocott et al., 2011). Moreover, it has been shown that lens develop from the preplacodal ectoderm (PE), requiring the sequential activation of a variety of transcription factors (Ogino et al., 2012). A combination of FGF signal with Wnt inhibition promotes preplacodal development (Streit, 2007). Xenopus FGF8 is expressed in the placodal region and anterior neural ridge at the neurula stages (Fletcher et al., 2006). Our analysis revealed that FGF8a can induce the expression of XAWP1 in naïve ectodermal tissue (Fig. 1). Taken together, these results suggest that FGF signal may be responsible for the expression of XAWP1 in the preplacodal ectoderm. XAWP1 is also detected in developing neural ectoderm. This expression pattern of XAWP1, in concert with its assumed mediation of Wnt signaling in the underlying mesoderm, suggests a possibility that it might affect neural induction and/or patterning. Indeed, depletion of XAWP1 was found to expand the expression of a pan-neural marker, Sox2 and a forebrain marker, Otx2 but to repress that of a hindbrain marker, Krox20 (data not shown). Neural crest induction is independent of the anterior-posterior patterning of the neural tissue (Wu et al., 2005). Therefore, XAWP1 is likely to affect these two processes separately.

XAWP1 activity is required for NC induction by Wnt8 or FGF8a ligand (Fig. 5). Notably, it acts upstream of the $\beta$-catenin/Tcf3 complex to control NC induction. Given that FGF8a induces the NC indirectly by activating the expression of Wnt8 (Hong et al., 2008), it seems likely that XAWP1 mediates both Wnt and FGF induction of the NC by affecting the Wnt signaling pathway. Supportingly, the depletion of AWP1 impairs the stability of $\beta$-catenin and the activity of a Wnt-responsive reporter gene, indicating its possible role as a Wnt signaling component. However, there is also evidence that Wnt and FGF signals act in parallel to regulate NC specification (Monsoro-Burq et al., 2005). Thus, it is possible that these two signals converge on AWP1 activity during NC induction. Moreover, FGF induction of AWP1 expression (Fig. 1) suggests that it might have a role in mediating the crosstalk between the Wnt and FGF signals in NC formation.

It appears that the mechanism underlying the activity of AWP1 in the Wnt signaling pathway involves the control of $\beta$-catenin stability as described above. It is well known that GSK3 and CK1-phosphorylated $\beta$-catenin undergoes $\beta$-Trcp-dependent ubiquitination for proteasome degradation (MacDonald et al., 2009). Since AWP1 has been shown to mediate polyubiquitination of proteins (Fenner et al., 2009), it is tempting to speculate that it could somehow affect the ubiquitination-mediated degradation of the $\beta$-catenin protein. In addition, AWP1 is implicated in the regulation of NF-kB activity (Chang et al., 2011). A recent study has shown that $\beta$-catenin acts as a transcriptional co-activator of NF-kB-dependent transcription (Armstrong et al., 2012). Moreover, NF-KB signaling is also involved in the specification of mouse mesencephalic NC cells (Fujita et al., 2011). Given these findings, it is possible that the loss-of-function of AWP1 interferes with the cooperation between Wnt and NF-KB signaling, which could lead to the inhibition of NC induction by Wnt signaling. In addition, it has been shown that a balance between the anti-apoptotic and apoptotic activities in the neural fold plays a critical role in the proper formation of the NC (Tribulo et al., 2004). Interestingly, the level of AWP1 expression affects the incidence of apoptotic cell death by regulating the activity of NF-KB (Chang et al., 2011). We also observed that both gain- and loss-of-function of $X A W P 1$ could increase the rate of apoptotic cell death in neural 
tissue (data not shown). Thus, the effects of XAWP1 on apoptotic cell death could be relevant to its regulation of NC specification. Further experiments will be necessary to test these hypotheses and to elucidate the precise mechanism underlying the activity of $A W P 1$.

\section{Materials and Methods}

\section{Embryo manipulation and lineage tracing}

In vitro fertilization, embryo culture and microinjection were carried out as described previously (Sive et al., 1989). The developmental stages of the embryos were determined according to the Nieuwkoop and Faber's normal table of development (Nieuwkoop and Faber, 1994). For lineage tracing, $\beta$-galactosidase mRNA ( $L a c Z, 50$ pg) was co-injected with MOs and other RNAs, and its activity was visualized with the X-Gal substrate (Duchefa Biochemie).

\section{Plasmid constructs, MOs and RNA synthesis}

The complete coding region of Xenopus AWP1 (GeneBank Accession No. BC042359) was amplified by PCR with the primers, 5'-ATGATTATGGCGCAGGAG-3' (forward) and 5'-TTATATCTTCTGAATCTTTTCTCC-3' (reverse) and then subcloned into a pGEM T vector (XAWP1-T). The DNA fragment, which was produced by digesting the XAWP1-T construct with $E c o R I$ and Spel restriction enzymes, was inserted into the EcoRII $X$ bal sites of a pCS2 vector (XAWP1-CS2+). The PCR product encompassing the coding region and $M O$ target sites was generated with the primers, 5'-CGGGATCCCGGCTACGGTTCCCGTTGCATG-3' (forward) and 5'- CGCGGATCCGCGTATCTTCTGAATCTTTTCTC-3' (reverse) and then inserted into the $B a m H I$ site of a pCS2+-Myc vector to produce a XAWP1-Myc construct. For sense mRNA synthesis, XAWP1-CS2+ and XAWP1-Myc constructs were linearized with Notl and transcribed with Sp6 RNA polymerase. The coding region of human AWP1 (GeneBank Accession No. AJ251095) was amplified by PCR with the primers, 5'- GGAATTCCATGGCTCAAGAAACTAAT-3' (forward) and 5'-TGCTCTAGAGCTCAAATCTTTTGGATCTT-3' (reverse) and inserted into the EcoRI/Xbal sites of a pCS2+-Myc vector to generate the hAWP1-Myc construct. Capped mRNAs were in vitro synthesized with the mMessage mMachine kit (Ambion). Anti-sense MOs were purchased from Gene Tools. XAWP1MOs had the following sequences: MO1, 5'-TTTGTCTCCTGCGCCATAATCATCC-3'; MO2, 5'-TCCACCATGCAACGGGAACCGTAGC-3'. The control MO was a standard MO from Gene Tools with the sequence 5'-CCTCTTACCTCAGTTACAATTTATA-3'.

\section{In situ hybridization}

Whole-mount in situ hybridization was performed as described previously (Harland, 1991). Anti-sense RNA probes were in vitro synthesized with digoxigenin-labeled nucleotides. An anti-sense XAWP1 probe was generated by transcribing the Spel-linearized XAWP1-T construct with T7 RNA polymerase. BM purple (Roche) was used as a substrate for the alkaline phosphatase.

\section{Western blotting}

Animal cap tissues were homogenized in Triton X-100 lysis buffer (20 $\mathrm{mM}$ Tris- $\mathrm{HCl}, 1 \%$ Triton X-100, $140 \mathrm{mM} \mathrm{NaCl}, 10 \%$ glycerol, 1 mM EGTA, $1.5 \mathrm{mM} \mathrm{MgCl}$, $1 \mathrm{mM}$ DTT, $1 \mathrm{mM}$ sodium orthovanadate, $50 \mathrm{mM} \mathrm{NaF}$, $10 \mu \mathrm{g} / \mathrm{ml}$ aprotinin, $10 \mu \mathrm{g} / \mathrm{ml}$ leupeptin). Equal amounts of protein were separated by $10 \%$ SDS-PAGE. Western blotting was carried out according to a standard protocol with anti-Myc (1:1000, Santa Cruz) and anti- $\beta$-actin (1:1000, Santa Cruz) antibodies.

\section{$R T-P C R$}

For RT-PCR analysis, total RNA was extracted from whole embryos and animal cap explants with TRI Reagent (Molecular Research Center) and treated with RNase-free DNase I (Roche Molecular Biochemicals) to remove genomic DNA. RNA was transcribed with M-MLV reverse transcriptase
(Promega) at $37^{\circ} \mathrm{C}$ for 1 hour. PCR products were analyzed on $2 \%$ agarose gels. The numbers of PCR cycles for each primer set were determined empirically to maintain amplification in the linear range. The sequences of the PCR primers used are as follows: XAWP1, 5'-TACACAGGCCAGCTCGTT GCC-3' (forward), 5'-AACGGTGAGTCCCGCAGAATACG-3'(reverse); Slug (Mizuseki et al., 1998); Sox9 (Monsoro-Burq et al., 2003); Pax3 (de Croze et al., 2011); and Msx1 (Suzuki et al., 1997).

\section{Histology and cartilage staining}

For histology, embryos were fixed in MEMFA at stage 40 and embedded in Paraplast (Leica). Sections were cut at a thickness of $10 \mu \mathrm{m}$ on a rotary microtome and stained with eosin (Sigma). For cartilage staining, embryos were fixed in MEMFA at stage 45, dehydrated in ethanol and stained for three nights in $0.04 \%$ alcian blue $/ 30 \%$ acetic acid in ethanol. Embryos were then washed extensively with ethanol and rehydrated in $2 \% \mathrm{KOH}$ solution. Finally, the embryos were washed in $20 \%$ glycerol $/ 2 \% \mathrm{KOH}$ for at least 1 hour and dehydrated through a glycerol series into $80 \%$ glycerol. Cranial cartilage was manually dissected out and photographed.

\section{Luciferase reporter assay}

For the TOPflash reporter assay, HEK 293T cells were transfected in 12-well dishes with Lipofectamine (Invitrogen). Approximately 48 hours after siRNA transfection, each well received $50 \mathrm{ng}$ of TOPflash reporter plasmid (Upstate) and $1 \mathrm{ng}$ of pRL-TK Renilla vector (as an internal control). Cells were lysed 24 hours after plasmid transfection and luciferase activity was measured with a dual luciferase assay system (Promega).

\section{Acknowledgements}

This work was supported by the National Research Foundation of Korea(NRF) MRC grant funded by the Korea government (MSIP)(20080062286).

\section{References}

ABU-ELMAGD, M., GARCIA-MORALES, C. and WHEELER, G.N. (2006). Frizzled7 mediates canonical Wnt signaling in neural crest induction. Dev Biol298:285-298.

ARMSTRONG, N.J., FAGOTTO, F., PROTHMANN, C. and RUPP, R.A. (2012). Maternal Wnt/beta-catenin signaling coactivates transcription through NF-kappaB binding sites during Xenopus axis formation. PLoS One 7: e36136.

CHANG, C. and HEMMATI-BRIVANLOU, A. (1998). Neural crest induction by Xwnt7B in Xenopus. Dev Biol 194: 129-134.

CHANG, E.J., HA, J., KANG, S.S., LEE, Z.H. and KIM, H.H. (2011). AWP1 binds to tumor necrosis factor receptor-associated factor 2 (TRAF2) and is involved in TRAF2-mediated nuclear factor-kappaB signaling. Int J Biochem Cell Biol 43 1612-1620.

DE CROZE, N., MACZKOWIAK, F. and MONSORO-BURQ, A.H. (2011). Reiterative AP2a activity controls sequential steps in the neural crest gene regulatory network. Proc Natl Acad Sci USA 108: 155-160.

DUAN, W., SUN, B., LI, T.W., TAN, B.J., LEE, M.K. and TEO, T.S. (2000). Cloning and characterization of AWP1, a novel protein that associates with serine/threonine kinase PRK1 in vivo. Gene 256: 113-121.

ELKOUBY, Y.M., ELIAS, S., CASEY, E.S., BLYTHE, S.A., TSABAR, N., KLEIN, P.S ROOT, H., LIU, K.J. and FRANK, D. (2010). Mesodermal Wnt signaling organizes the neural plate via Meis3. Development 137: 1531-1541.

FENNER, B.J., SCANNELL, M. and PREHN, J.H. (2009). Identification of polyubiquitin binding proteins involved in NF-kappaB signaling using protein arrays. Biochim Biophys Acta 1794: 1010-1016.

FLETCHER, R.B., BAKER, J.C. and HARLAND, R.M. (2006). FGF8 spliceforms mediate early mesoderm and posterior neural tissue formation in Xenopus. Development 133: 1703-1714.

FUJITA, K., YASUI, S., SHINOHARA, T. and ITO, K. (2011). Interaction between NFkappaB signaling and Notch signaling in gliogenesis of mouse mesencephalic neural crest cells. Mech Dev 128: 496-509.

GARCIA-CASTRO, M.I., MARCELLE, C. and BRONNER-FRASER, M. (2002). Ecto- 
dermal Wnt function as a neural crest inducer. Science 297: 848-851.

GROCOTT, T., JOHNSON, S., BAILEY, A.P. and STREIT, A. (2011). Neural crest cells organize the eye via TGF-beta and canonical Wnt signalling. Nat Commun 2: 265.

HARLAND, R.M. (1991). In situ hybridization: an improved whole-mount method for Xenopus embryos. Methods Cell Biol 36: 685-695.

HASSLER, C., CRUCIAT, C.M., HUANG, Y.L., KURIYAMA, S., MAYOR, R. and NIEHRS, C. (2007). Kremen is required for neural crest induction in Xenopus and promotes LRP6-mediated Wnt signaling. Development 134: 4255-4263.

HEEG-TRUESDELL, E. and LABONNE, C. (2004). A slug, a fox, a pair of sox: transcriptional responses to neural crest inducing signals. Birth Defects Res $C$ Embryo Today 72: 124-139.

HONG, C.S., PARK, B.Y. and SAINT-JEANNET, J.P. (2008). Fgf8a induces neural crest indirectly through the activation of Wnt8 in the paraxial mesoderm. Development 135: 3903-3910.

IKEYA, M., LEE, S.M., JOHNSON, J.E., MCMAHON, A.P. and TAKADA, S. (1997). Wnt signalling required for expansion of neural crest and CNS progenitors. Nature 389: 966-970

KIMELMAN, D. (2006). Mesoderm induction: from caps to chips. Nat Rev Genet 7: 360-372.

LE DOUARIN, N.M. and DUPIN, E. (2003). Multipotentiality of the neural crest. Curr Opin Genet Dev 13: 529-536.

LEWIS, J.L., BONNER, J., MODRELL, M., RAGLAND, J.W., MOON, R.T., DORSKY, R.I. and RAIBLE, D.W. (2004). Reiterated Wnt signaling during zebrafish neural crest development. Development 131: 1299-1308.

MACDONALD, B.T., TAMAI, K. and HE, X. (2009). Wnt/beta-catenin signaling: components, mechanisms, and diseases. Dev Cell 17: 9-26.

MIYATA, N., OKUMOTO, K., MUKAI, S., NOGUCHI, M. and FUJIKI, Y. (2012). AWP1/ ZFAND6 functions in Pex5 export by interacting with cys-monoubiquitinated Pex5 and Pex6 AAA ATPase. Traffic 13: 168-183.

MIZUSEKI, K., KISHI, M., MATSUI, M., NAKANISHI, S. and SASAI, Y. (1998). Xenopus Zic-related-1 and Sox-2, two factors induced by chordin, have distinct activities in the initiation of neural induction. Development 125: 579-587.

MONSORO-BURQ, A.H., FLETCHER, R.B. and HARLAND, R.M. (2003). Neural crest induction by paraxial mesoderm in Xenopus embryos requires FGF signals. Development 130: 3111-3124.

MONSORO-BURQ, A.H., WANG, E. and HARLAND, R. (2005). Msx1 and Pax3 cooperate to mediate FGF8 and WNT signals during Xenopus neural crest induction. Dev Cell 8: 167-178.

NIEUWKOOP, P.D. and FABER, J. (1994). Normal table of Xenopus laevis (Daudin): a systematical and chronological survey of the development from the fertilized egg till the end of metamorphosis. Garland Pub., New York.

OGINO, H., OCHI, H., REZA, H.M. and YASUDA, K. (2012). Transcription factors involved in lens development from the preplacodal ectoderm. Dev Bio/363:333-347.

PRASAD, M.S., SAUKA-SPENGLER, T. and LABONNE, C. (2012). Induction of the neural crest state: control of stem cell attributes by gene regulatory, posttranscriptional and epigenetic interactions. Dev Biol 366: 10-21.

SAUKA-SPENGLER, T. and BRONNER-FRASER, M. (2008). A gene regulatory network orchestrates neural crest formation. Nat Rev Mol Cell Biol 9: 557-568.

SIVE, H.L., HATTORI, K. and WEINTRAUB, H. (1989). Progressive determination during formation of the anteroposterior axis in Xenopus laevis. Cell 58: 171-180.

STEVENTON, B., CARMONA-FONTAINE, C. and MAYOR, R. (2005). Genetic network during neural crest induction: from cell specification to cell survival. Semin Cell Dev Biol 16: 647-654

STREIT, A. (2007). The preplacodal region: an ectodermal domain with multipotential progenitors that contribute to sense organs and cranial sensory ganglia. Int $J$ Dev Biol 51: 447-461.

STUHLMILLER, T.J. and GARCIA-CASTRO, M.I. (2012). Current perspectives of the signaling pathways directing neural crest induction. Cell Mol Life Sci69:3715-3737.

SUZUKI,A., UENO, N. and HEMMATI-BRIVANLOU,A. (1997). Xenopusms 1 mediates epidermal induction and neural inhibition by BMP4. Development 124:3037-3044.

TAMAI, K., SEMENOV, M., KATO, Y., SPOKONY, R., LIU, C., KATSUYAMA, Y., HESS, F., SAINT-JEANNET, J.P. and HE, X. (2000). LDL-receptor-related proteins in Wnt signal transduction. Nature 407: 530-535.

THEVENEAU, E. and MAYOR, R. (2012). Neural crest delamination and migration: from epithelium-to-mesenchyme transition to collective cell migration. Dev Bio 366: 34-54.

TRIBULO, C., AYBAR, M.J., SANCHEZ, S.S. and MAYOR, R. (2004). A balance between the anti-apoptotic activity of Slug and the apoptotic activity of msx1 is required for the proper development of the neural crest. Dev Biol 275: 325-342.

WU, J., YANG, J. and KLEIN, P.S. (2005). Neural crest induction by the canonical Wnt pathway can be dissociated from anterior-posterior neural patterning in Xenopus. Dev Biol 279: 220-232. 


\section{Further Related Reading, published previously in the Int. J. Dev. Biol.}

Developmental expression and regulation of the chemokine CXCL14 in Xenopus

Byung-Yong Park, Chang-Soo Hong, Faraz A. Sohail and Jean-Pierre Saint-Jeannet

Int. J. Dev. Biol. (2009) 53: 535-540

http://dx.doi.org/10.1387/ijdb.092855bp

Msx1 and Msx2 have shared essential functions in neural crest but may be dispensable in epidermis and axis formation in Xenopus

Deepak Khadka, Ting Luo and Thomas D. Sargent

Int. J. Dev. Biol. (2006) 50: 499-502

http://dx.doi.org/10.1387/ijdb.052115dk

Early stages of neural crest ontogeny: formation and regulation of cell delamination Chaya Kalcheim and Tal Burstyn-Cohen

Int. J. Dev. Biol. (2005) 49: 105-116

http://dx.doi.org/10.1387/ijdb.041949ck

Origins and plasticity of neural crest cells and their roles in jaw and craniofacial evolution Paul A Trainor, Kristin R Melton and Miguel Manzanares

Int. J. Dev. Biol. (2003) 47: 541-553

http://www.intjdevbiol.com/web/paper/14756330

Xerl, a novel CNS-specific secretory protein, establishes the boundary between neural plate and neural crest

S Kuriyama and T Kinoshita

Int. J. Dev. Biol. (2001) 45: 845-852

http://www.intjdevbiol.com/web/paper/11804027

5 yr ISI Impact Factor $(2011)=2.959$

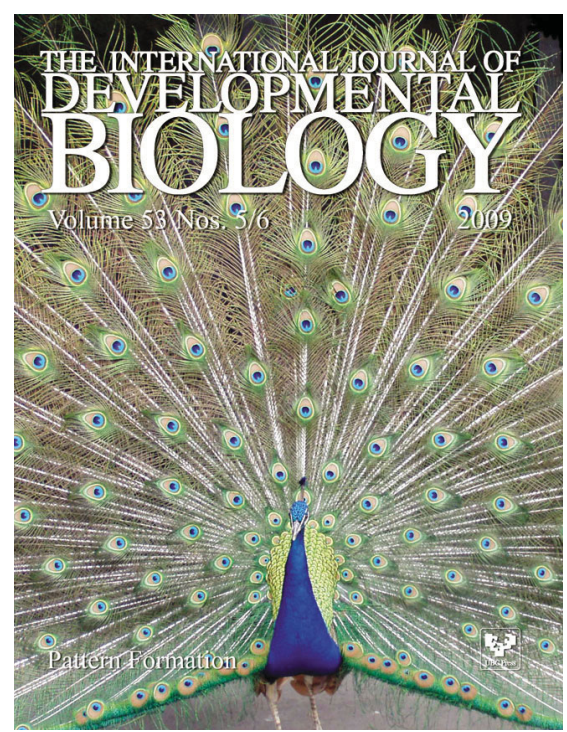

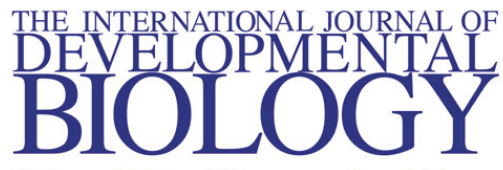

Volume 48 Nos. $5 / 6$

Special issue

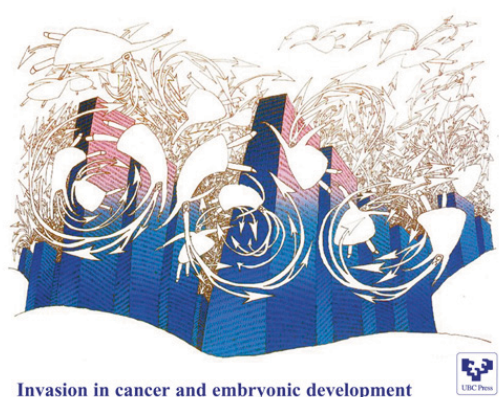

Invasion in cancer and embryonic development
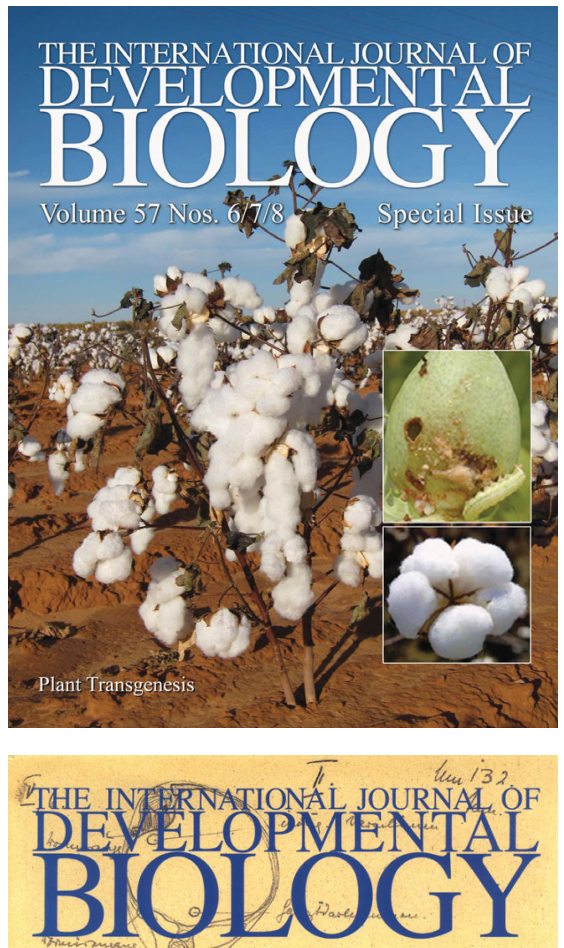

Volume 45 NO. 1

Special issue

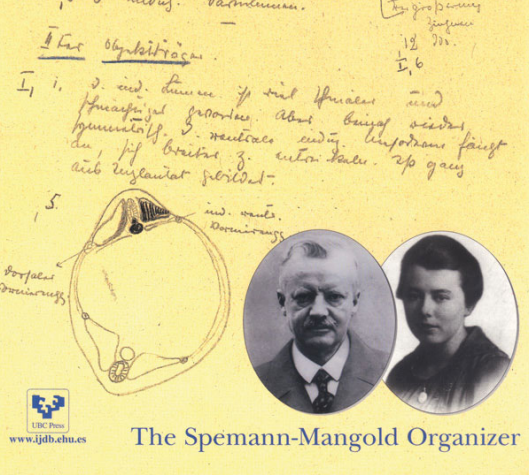

